\title{
Comparison of Complications between Gender during Spinal Anesthesia
}

\author{
Vahabi $S^{1}$, Karimi $\mathrm{A}^{1^{*}}$ and Ghanavati $\mathrm{M}^{2}$ \\ ${ }^{1}$ Faculty of Medicine, Department of Anesthesiology, Lorestan University of Medical Sciences, Khorramabad, Iran \\ ${ }^{2}$ Student of Research Committee, Lorestan University of Medical Sciences, Khorramabad, Iran
}
${ }^{*}$ Corresponding author: Karimi A, Assistant Professor, Department of Anesthesiology, Lorestan University of Medical Sciences, Khorramabad, Iran, E-mail: dr.a.karimi53@gmail.com

Citation: Vahabi S, Karimi A, Ghanavati M (2018) Comparison of Complications between Gender during Spinal Anesthesia. J Surg Oper Care 3(2): 201. doi: 10.15744/2455-7617.3.201

Received Date: January 18, 2018 Accepted Date: June 29, 2018 Published Date: July 2, 2018

\begin{abstract}
Background: The side effects of spinal anesthesia has been the focus of many researchers. These complications can affect patients' satisfaction following surgery and duration of hospitalization. Therefore, factors that lead to increased risk of side effects must be identified, one of these factors is gender. This study evaluated the relationship between gender and adverse effects after spinal anesthesia.

Materials and Methods: In this cohort study, 1200 patients including 600 men and 600 women underwent spinal anesthesia with 5\% lidocaine injected into L3-4 or L4-5interspace with the patient in either sitting or lateral position. The subjects include patients scheduled for lower abdominal site and orthopedic surgeries (leg to the bottom), analysis was done at 6,12, 18, and 24 hours after recovery, and some variables in the second and third days were also analyed. To statistically analyze the side effects of spinal anesthesia, chi-square test was used.

Result: Result showed that nausea, vomiting, headache, urinary retention (in recovery unit) and back pain (18hours) were statistically significantly different $(\mathrm{P}<0.05)$. There was no significant difference between the incidence of other complications and gender.

Conclusion: According to this study, gender can lead to differences in the incidence of some complications after spinal anesthesia.

Keywords: Spinal anesthesia; Gender; Adverse effects; Bradypnae; Tachycardia; Hypertension; Hypotension
\end{abstract}

\section{Introduction}

Spinal anesthesia involves the induction of reversible motor function and sensation loss into the subarachnoid space using small amounts of local anesthetic [1]. This anesthesia provider places the needle in the adult patient in a position below L2 to avoid spinal cord trauma [2]. It gave a perfect condition of operation for: surgical protocol below the umbilicus, gynecologic/obstetric procedures of uterus and perineum, hernia repairs, genitourinary procedures, orthopedic procedures from the hip downward [3]. Patients with severe dehydration or hypovolemic should not be subjected to spinal anesthesia[4]. Patients under spinal anesthetic should be preloaded with 1-1.5 liters of a crystalloid solution, such as ringers lactate, immediately prior to the block [5]. Advantages of this type of anesthesia include; easy to perform, reliable, gave good operating conditions for the surgeon, it is inexpensive than general anesthesia, normal gastrointestinal function returns faster with spinal anesthesia [6-8]. Patients maintain a patent airway, a decrease in pulmonary complications compared to general anesthesia, and a decreased incidence of deep vein thrombosis and pulmonary embolism formation compared to general anesthesia [9].The side effects of spinal anesthesia have recently been the area of interest [10]. These complications can affect patients' satisfaction following surgery and duration of hospitalization [11]. Therefore, factors that lead to increased risk side effects must be identified for better patients management after surgery, one of these factors is gender [12]. This study evaluated the relationship between gender and adverse effects including nausea, vomiting, shivering, agitation, tachycardia, bradycardia, hypotension, hypertension, complete spinal block, surgical site pain, bradypnae, headache, back pain, pain during injection of anesthetic, urinary retention, hypothermia, drowsiness and subdural hematoma after spinal anesthesia.

\section{Materials and Methods}

This study was carried out at Lorestan University of Medical Sciences (LUMS) teaching hospital, Khoramabad, Iran. In this cohort study, 1200 patients including 600 men and 600 women who went through spinal anesthesia were examined (Table 1). The subject are grouped into two, 600 were administered with 5\% lidocaine which was injected into L3-4 or L4-5 interspace with the patient in either sitting or lateral position. The subjects include patients scheduled for lower abdominal site and orthopedic surgeries (leg to the bottom), analysis was done at $6,12,18$, and 24 hours after recovery, and some variables in the second and third days were 
examined. Amongst the women, $286(47.7 \%)$ patients underwent lower abdominal surgery and 314 (53.2\%) patients underwent orthopedic surgery. Amongst the men, 224 (37.3\%) patients underwent lower abdominal surgery and 367 (62.7\%) patients underwent orthopedic surgery. Eligible patients were enrolled, and a written informed consent was obtained prior to the study. Inclusion criteria in the study was the possibility of surgery with spinal anesthesia, ASA CLASS I, the absence of any systemic disease and aged between 18 to 65 years as to the type of surgery. Patients who went through orthopedic surgery of the lower abdomen, and legs down to the bottom were selected. The combination of patients by age and type of surgery was also performed. In this study, before the onset of spinal anesthesia, systolic blood pressure (SBP), heart rate (HR), respiratory rate (RR) and body temperature were measured. The first spinal anesthesia was administered with catheter to the vessel where 18 and $20 \mathrm{cc} / \mathrm{Kg}$ of ringer lactated solution was received. Spinal anesthesia was performed with 5\% lidocaine injected into L3-4 or L4-5 interspace with the patient in either sitting or lateral position, this standard procedure was performed by an anesthesiologist. Patients with more than one attempt of spinal anesthesia were excluded.

\begin{tabular}{|c|c|c|c|}
\hline Group & $\begin{array}{c}\text { The frequency of lower } \\
\text { abdominal surgery }\end{array}$ & $\begin{array}{c}\text { The frequency of } \\
\text { orthopedic surgery }\end{array}$ & $\begin{array}{c}\text { Total Number } \\
\text { (percent) }\end{array}$ \\
\hline Men & $224(37.3) \%$ & $376(62.7) \%$ & $600(100 \%)$ \\
\hline Women & $314(52.3) \%$ & $286(47.7) \%$ & $600(100 \%)$ \\
\hline \multicolumn{3}{r}{ Table 1: Distribution of surgical procedures by gender } \\
Thy
\end{tabular}

Eligible patients at the time of recovery after surgery, at 6,12, 18 and 24hours and also on the second and third postoperative complications were followed by dialogue through questionnaires (Table 2). Patients with hypertension complication had $20 \%$ increase in SBP. Lower body temperature of $36.5^{\circ} \mathrm{C}$ was considered as hypothermia. HR of 100 beats per minute, $\leq 55, \geq 55$

\begin{tabular}{|c|c|c|c|c|c|}
\hline Complications & Group & Present (percent) & $\begin{array}{l}\text { Absent (per- } \\
\text { cent) }\end{array}$ & $\begin{array}{l}\text { Total Number } \\
\text { (percent) }\end{array}$ & $\mathrm{P}$-value \\
\hline Hypertension & $\begin{array}{c}\text { Male } \\
\text { Female } \\
\text { Total }\end{array}$ & $\begin{array}{c}19(3.2 \%) \\
30(5 \%) \\
49(4.1 \%)\end{array}$ & $\begin{array}{c}581(96.8 \%) \\
570(95 \%) \\
1101(95.9 \%)\end{array}$ & $\begin{array}{c}600(100 \%) \\
600(100 \%) \\
1200(100 \%)\end{array}$ & 0.109 \\
\hline Hypotension & $\begin{array}{c}\text { Male } \\
\text { Female } \\
\text { Total }\end{array}$ & $\begin{array}{c}13(2.2 \%) \\
12(2 \%) \\
25(2.1 \%)\end{array}$ & $\begin{array}{c}587(97.8 \%) \\
588(98 \%) \\
1175(97.9 \%)\end{array}$ & $\begin{array}{c}600(100 \%) \\
600(100 \%) \\
1200(100 \%)\end{array}$ & 0.840 \\
\hline Tachycardia & $\begin{array}{c}\text { Male } \\
\text { Female } \\
\text { Total }\end{array}$ & $\begin{array}{c}22(3.7 \%) \\
26(4.3 \%) \\
48(4 \%)\end{array}$ & $\begin{array}{l}578(96.3 \%) \\
574(95.7 \%) \\
1102(96 \%)\end{array}$ & $\begin{array}{c}600(100 \%) \\
600(100 \%) \\
1200(100 \%)\end{array}$ & 0.555 \\
\hline Bradycardia & $\begin{array}{c}\text { Male } \\
\text { Female } \\
\text { Total }\end{array}$ & $\begin{array}{c}20(3.3 \%) \\
16(2.7 \%) \\
36(3 \%)\end{array}$ & $\begin{array}{c}580(96.7 \%) \\
584(97.3 \%) \\
1164(97 \%)\end{array}$ & $\begin{array}{c}600(100 \%) \\
600(100 \%) \\
1200(100 \%)\end{array}$ & 0.498 \\
\hline Nausea & $\begin{array}{c}\text { Male } \\
\text { Female } \\
\text { Total }\end{array}$ & $\begin{array}{c}57(9.5 \%) \\
91(15.2 \%) \\
148(12.3 \%)\end{array}$ & $\begin{array}{c}543(90.5 \%) \\
509(84.8 \%) \\
1052(87.7 \%)\end{array}$ & $\begin{array}{c}600(100 \%) \\
600(100 \%) \\
1200(100 \%)\end{array}$ & 0.003 \\
\hline TNS & $\begin{array}{c}\text { Male } \\
\text { Female } \\
\text { Total }\end{array}$ & $\begin{array}{l}4(0.7 \%) \\
4(0.7 \%) \\
8(0.7 \%)\end{array}$ & $\begin{array}{c}596(99.3 \%) \\
596(99.3 \%) \\
1192(99.3 \%)\end{array}$ & $\begin{array}{c}600(100 \%) \\
600(100 \%) \\
1200(100 \%)\end{array}$ & 1.000 \\
\hline Shriving & $\begin{array}{c}\text { Male } \\
\text { Female } \\
\text { Total }\end{array}$ & $\begin{array}{l}24(4 \%) \\
36(6 \%) \\
60(5 \%)\end{array}$ & $\begin{array}{c}576(96 \%) \\
564(94 \%) \\
1160(95 \%)\end{array}$ & $\begin{array}{c}600(100 \%) \\
600(100 \%) \\
1200(100 \%)\end{array}$ & 0.112 \\
\hline back ache & $\begin{array}{c}\text { Male } \\
\text { Female } \\
\text { Total }\end{array}$ & $\begin{array}{c}7(1.2 \%) \\
14(2.3 \%) \\
21(1.8 \%)\end{array}$ & $\begin{array}{c}593(98.8 \%) \\
586(97.7 \%) \\
1179(98.2 \%)\end{array}$ & $\begin{array}{c}600(100 \%) \\
600(100 \%) \\
1200(100 \%)\end{array}$ & 0.313 \\
\hline Drowsiness & $\begin{array}{c}\text { Male } \\
\text { Female } \\
\text { Total }\end{array}$ & $\begin{array}{c}21(3.5 \%) \\
15(2.5 \%) \\
36(3 \%)\end{array}$ & $\begin{array}{c}579(96.5 \%) \\
585(97.5 \%) \\
1164(97 \%)\end{array}$ & $\begin{array}{c}600(100 \%) \\
600(100 \%) \\
1200(100 \%)\end{array}$ & 0.310 \\
\hline Fidget & $\begin{array}{c}\text { Male } \\
\text { Female } \\
\text { Total }\end{array}$ & $\begin{array}{c}8(1.3 \%) \\
10(1.7 \%) \\
18(1.5 \%)\end{array}$ & $\begin{array}{c}592(98.7 \%) \\
590(98.3 \%) \\
1182(98.5 \%)\end{array}$ & $\begin{array}{c}600(100 \%) \\
600(100 \%) \\
1200(100 \%)\end{array}$ & 0.635 \\
\hline Urinary retention & $\begin{array}{c}\text { Male } \\
\text { Female } \\
\text { Total }\end{array}$ & $\begin{array}{c}73(12.2 \%) \\
60(10 \%) \\
133(11.1 \%)\end{array}$ & $\begin{array}{c}528(87.8 \%) \\
540(90 \%) \\
1067(88.9 \%)\end{array}$ & $\begin{array}{c}600(100 \%) \\
600(100 \%) \\
1200(100 \%)\end{array}$ & 0.232 \\
\hline Hypothermia & $\begin{array}{c}\text { Male } \\
\text { Female } \\
\text { Total }\end{array}$ & $\begin{array}{l}19(3.2 \%) \\
20(3.3 \%) \\
39(3.2 \%)\end{array}$ & $\begin{array}{c}581(96.8 \%) \\
580(96.7 \%) \\
1161(96.8 \%)\end{array}$ & $\begin{array}{c}600(100 \%) \\
600(100 \%) \\
1200(100 \%)\end{array}$ & 0.871 \\
\hline Bradypoenea & $\begin{array}{l}\text { Male } \\
\text { Female } \\
\text { Total }\end{array}$ & $\begin{array}{c}3(0.5 \%) \\
6(1 \%) \\
9(0.8 \%)\end{array}$ & $\begin{array}{c}597(99.5 \%) \\
594(99 \%) \\
1191(99.2 \%)\end{array}$ & $\begin{array}{c}600(100 \%) \\
600(100 \%) \\
1200(100 \%)\end{array}$ & 0.315 \\
\hline
\end{tabular}

Table 2: Distribution of subjects based on the incidence of different parameters at different times based on gender 
beats/mins, were for tachycardia and bradycardia respectively. A smaller RR of $12 / \mathrm{mins}$ for bradypnae. In the study, inability to retain urine or bladder without volume of $600 \mathrm{~mL}$ was indicative of the occurrence of this complication. Nausea, and epigastric discomfort in the stomach,the ratings of the severity of this complication include: no nausea, (0), mild nausea and tolerable (1), annoying nausea (2). Vomiting of stomach contents as well as withdrawal was also evaluated, scoring include: no vomiting (0), 1 or 2 times vomiting (1) and more than 2 turns of vomiting (2). The feelings of pain at surgical site ratings were done using the Visual Analogue Scale (VAS) scores based on the Verbal Descriptor Scale (VDS), these include; painless (0), brief pain (1$3)$, moderate pain $(4-7)$, pain $(8,9)$ and very severe and unbearable pain $(10)$. Headache, especially in the frontal and occipital region were rated as; no headaches $(0)$, headache $(1-3)$, moderate headache $(4-7)$, headache $(8,9)$ and severe and unbearable headache (10). The headache gets worse with walking and sitting, lying down is better. It can also bring about nausea and vomiting. These complications may occur 24 hours after surgery, so patients were followed in second and third days. Transient neurological disorder as well as any dizzy feeling, numbness in the buttock and lower extremities were studied. Since these side effects may occur after 24 hours, patients were followed in second and third day. Restlessness was also felt and the need was irresistible, mental instability and movement. The patient felt pain during anesthesia because of the liquid administered on the lower limbs. A T4 segment sensory level, above which is complication during spinal anesthesia, hypotension and bradycardia were also examined. Shivering and vibratory motions of head, and limbs were evaluated. Back pain in the lumbar spine after anesthesia was examined, this pain was rated on VAS at VDS scores graded as; painless (0), brief pain (1-3), moderate pain (3-7), pain (7-9) and very severe and unbearable pain (10). The need for patients' intervention were evaluated as well, if the surgical site pain score is greater than 4 , treatment with pethidine injection $1 \mathrm{mg} / \mathrm{kg}$ was allowed. In the case of post spinal headache, $50 \mathrm{mg}$ diclofenac for patients below $50 \mathrm{~kg}$, and $100 \mathrm{mg}$ for patients greater than $50 \mathrm{~kg}$ was used. If nausea and vomiting occurred, metoclopramide was administered, a score of more than 2 is assigned. In case of chills and muscle injection, $25 \mathrm{mg}$ pethidine was administered. In case of hypothermia, patients were capped with more blankets.

After sufficient control of pain, patients are regarded as ready in accordance with the Departmental protocol. Patients cardiopulmonary was stable and their spinal anesthesia level sensory residual was $<\mathrm{S} 1$.

\section{Statistical Analysis}

After collecting the questionnaires, data analysis of incidence between the two sexes were compared after spinal anesthesia. Statistical analysis was carried out using a multivariate regression analysis. Chi-square test was used to analyze the incidence of complications.

\section{Results}

In this cohort study where 600 patients were female and 600 were male. Analysis was done at 6, 12, 18, and 24hours after recovery, for some variables, such as headache and pain in the second and third days were studied. Table 3 shows the incidence of hypertension in men and women, which was 19 patients $(2.3 \%)$, and 30 (5\%) respectively, there was no significant difference was observed between incidence of hypertension and gender $(\mathrm{P}=0.109)$. The incidence of hypotension in men and women was 13 patients $(3.2 \%)$ and 12 patients $(2.2 \%)$ respectively, there was no significant difference between hypotension and gender $(\mathrm{P}=0.840)$ (Table 3). The incidence of tachycardia in men and women was 22 patients (3.7\%) and 26 patients (4.3\%) respectively, there was no significant difference between tachycardia and gender $(\mathrm{P}=0.555)$ (Table 3$)$. The incidence of bradycardia in men and women was 20 patients $(3.3 \%)$ and 16 patients $(2.7 \%)$ respectively, there was no statistically significantly difference between gender and bradycardia incidence $(\mathrm{P}=0.498)$ (Table 3$)$. The incidence of nausea in men and women was 57 patients $(9.5 \%)$ and $91(15.2 \%)$ respectively $(\mathrm{P}=0.004)$ (Table 3$)$. The incidence of vomiting in men and women was $34(6.3 \%)$, and 60 (10\%), also average vomiting in men and women was 5 patients $(0.8 \%)$ and 9 patients (1.5\%) (with severe vomiting) respectively, there was a statistically significantly difference observed between incidence of vomiting and gender $(\mathrm{P}=0.006)$ (Table 3$)$. The incidence of TNS in men and women was 4 patients $(0.7 \%)$ and 4 patients $(0.7 \%)$ respectively, there was no statistically significantly difference observed between TNS and gender $(\mathrm{P}=0.001)$ (Table 3$)$. The incidence of shivering in men and women was $24(4 \%)$ and $36(6 \%)$ respectively, there was no statistically significantly difference observed between shivering and gender $(\mathrm{P}=0.112)$ (Table 3$)$. The incidence of back pain in men and women was 7 patients $(1.2 \%)$ and $14(2.3 \%)$ respectively, there was a statistically significantly difference observed between back pain and gender $(\mathrm{P}=0.313)$, but, significant difference was observed 18 hours after surgery $(\mathrm{P}=0.014)($ Table 3$)$. The incidence of restlessness in men and women was $8(1.3 \%)$ and $10(1.7 \%)$ respectively, there was no statistically significantly difference observed between restlessness and gender $(\mathrm{P}=0.635)$ (Table 3$)$. The incidence of urinary retention in men and women was $73(12.2 \%)$, and $60(10 \%)$ respectively, there was a statistically significantly difference observed between urinary retention and gender $(\mathrm{P}<0.05)$ (Table 3). The incidence of hypothermia in men and women was $19(3.2 \%)$ and $20(3 / 3 \%)$ respectively, there was no statistically significantly difference observed between hypothermia and gender $(\mathrm{P}=0.871)$ (Table 3$)$. The incidence of bradypnae in men and women was $3(0.5 \%)$ and $6(1 \%)$, respectively, there was no statistically significantly difference observed between bradypnae and gender $(\mathrm{P}=0.315)$. The incidence of mild headache in men and women was $28(1.4 \%)$ and $60(2.10 \%)$, they score 1 and 3 respectively (Table 3$)$. The incidence of average headache in men and women was $9(1.5 \%)$ and $6(1 \%)$, they score between 4 and 7 respectively. The incidence of headache in men and women was $3(0.5 \%)$ and $11(1.8 \%)$, they score of 8 and 9 respectively, a significant different was observed between sex and headaches $(\mathrm{P}=0.245)$ (Table 4$)$. The incidence of mild pain in men and women was $26(4.3 \%)$ and $22(3.7 \%)$ respectively at the surgical site. The incidence of moderate pain in men and women was $4(0.7 \%)$ and $7(1.2 \%)$ respectively at surgical site. 


\begin{tabular}{|c|c|c|c|c|c|}
\hline \multicolumn{6}{|c|}{ Distribution of subjects based on the incidence of hypertension at different times based on gender } \\
\hline Time & Group & Present (percent) & Absent (percent) & $\begin{array}{l}\text { Total Number } \\
\text { (percent) }\end{array}$ & P-value \\
\hline Recovery & $\begin{array}{c}\text { Male } \\
\text { Female } \\
\text { Total }\end{array}$ & $\begin{array}{c}6(1 \%) \\
7(1.2 \%) \\
13(1.2 \%)\end{array}$ & $\begin{array}{c}59499 \\
59398.8 \\
118798.8\end{array}$ & $\begin{array}{c}600(100 \%) \\
600(100 \%) \\
1200(100 \%)\end{array}$ & 0.780 \\
\hline 6 hours & $\begin{array}{c}\text { Male } \\
\text { Female } \\
\text { Total }\end{array}$ & $\begin{array}{c}5(0.8 \%) \\
8(1.3 \%) \\
13(1.2 \%)\end{array}$ & $\begin{array}{c}59599.2 \\
59298.7 \\
118798.8\end{array}$ & $\begin{array}{c}600(100 \%) \\
600(100 \%) \\
1200(100 \%)\end{array}$ & 0.403 \\
\hline 12 hours & $\begin{array}{c}\text { Male } \\
\text { Female } \\
\text { Total }\end{array}$ & $\begin{array}{l}2(0.3 \%) \\
5(0.8 \%) \\
7(0.6 \%)\end{array}$ & $\begin{array}{c}59898.7 \\
59598.2 \\
119399.4\end{array}$ & $\begin{array}{c}600(100 \%) \\
600(100 \%) \\
1200(100 \%)\end{array}$ & 0.255 \\
\hline 18 hours & $\begin{array}{c}\text { Male } \\
\text { Female } \\
\text { Total }\end{array}$ & $\begin{array}{c}4(0.7 \%) \\
7(1.2 \%) \\
11(0.9 \%)\end{array}$ & $\begin{array}{c}59699.3 \\
59398.8 \\
118999.1\end{array}$ & $\begin{array}{c}600(100 \%) \\
600(100 \%) \\
1200(100 \%)\end{array}$ & 0.364 \\
\hline 24 hours & $\begin{array}{c}\text { Male } \\
\text { Female } \\
\text { Total }\end{array}$ & $\begin{array}{l}2(0.3 \%) \\
3(0.5 \%) \\
5(0.4 \%)\end{array}$ & $\begin{array}{c}59899.7 \\
59799.5 \\
119599.6\end{array}$ & $\begin{array}{c}600(100 \%) \\
600(100 \%) \\
1200(100 \%)\end{array}$ & 0.654 \\
\hline
\end{tabular}

\begin{tabular}{|c|c|c|c|c|c|}
\hline \multicolumn{6}{|c|}{ Distribution of subjects on the basis of Hypotension at different times based on gender } \\
\hline Recovery & $\begin{array}{c}\text { Male } \\
\text { Female } \\
\text { Total }\end{array}$ & $\begin{array}{c}9(1.5 \%) \\
8(1.3 \%) \\
17(1.4 \%)\end{array}$ & $\begin{array}{c}591(98.5 \%) \\
592(98.7 \%) \\
1183(98.6 \%)\end{array}$ & $\begin{array}{c}600(100 \%) \\
600(100 \%) \\
1200(100 \%)\end{array}$ & 0.807 \\
\hline 6 hours & $\begin{array}{c}\text { Male } \\
\text { Female } \\
\text { Total }\end{array}$ & $\begin{array}{l}3(0.5 \%) \\
2(0.3 \%) \\
5(0.4 \%)\end{array}$ & $\begin{array}{c}597(99 \%) \\
598(99.7) \\
1195(99.6) \\
\end{array}$ & $\begin{array}{c}600(100 \%) \\
600(100 \%) \\
1200(100 \%)\end{array}$ & 0.654 \\
\hline 12 hours & $\begin{array}{c}\text { Male } \\
\text { Female } \\
\text { Total }\end{array}$ & $\begin{array}{l}0(0 \%) \\
0(0 \%) \\
0(0 \%)\end{array}$ & $\begin{array}{l}0(0 \%) \\
0(0 \%) \\
0(0 \%)\end{array}$ & $\begin{array}{c}600(100 \%) \\
600(100 \%) \\
1200(100 \%)\end{array}$ & - \\
\hline 18 hours & $\begin{array}{c}\text { Male } \\
\text { Female } \\
\text { Total }\end{array}$ & $\begin{array}{l}1(0.2 \%) \\
2(0.3 \%) \\
3(0.2 \%)\end{array}$ & $\begin{array}{c}599(99.8 \%) \\
598(99.7 \%) \\
1197(99.8)\end{array}$ & $\begin{array}{c}600(100 \%) \\
600(100 \%) \\
1200(100 \%)\end{array}$ & 0.563 \\
\hline 24 hours & $\begin{array}{c}\text { Male } \\
\text { Female } \\
\text { Total }\end{array}$ & $\begin{array}{l}0(0 \%) \\
0(0 \%) \\
0(0 \%)\end{array}$ & $\begin{array}{l}0(0 \%) \\
0(0 \%) \\
0(0 \%)\end{array}$ & $\begin{array}{c}600(100 \%) \\
600(100 \%) \\
1200(100 \%)\end{array}$ & - \\
\hline \multicolumn{6}{|c|}{ Distribution of subjects based on the incidence of tachycardia at different times based on gender } \\
\hline Recovery & $\begin{array}{l}\text { Male } \\
\text { Female } \\
\text { Total }\end{array}$ & $\begin{array}{c}10(1.7 \%) \\
8(1.3 \%) \\
18(1.5 \%)\end{array}$ & $\begin{array}{c}590(98.3 \%) \\
592(98.7 \%) \\
1182(98.5 \%)\end{array}$ & $\begin{array}{c}600(100 \%) \\
600(100 \%) \\
1200(100 \%)\end{array}$ & 0.827 \\
\hline 6 hours & $\begin{array}{c}\text { Male } \\
\text { Female } \\
\text { Total }\end{array}$ & $\begin{array}{c}7(1.2 \%) \\
8(1.3 \%) \\
15(1.2 \%)\end{array}$ & $\begin{array}{c}593(98.8 \%) \\
592(98.7 \%) \\
1185(98.8 \%)\end{array}$ & $\begin{array}{c}600(100 \%) \\
600(100 \%) \\
1200(100 \%)\end{array}$ & 0.693 \\
\hline 12 hours & $\begin{array}{l}\text { Male } \\
\text { Female } \\
\text { Total }\end{array}$ & $\begin{array}{c}6(1 \%) \\
9(1.5 \%) \\
15(1.2 \%)\end{array}$ & $\begin{array}{c}594(99 \%) \\
591(98.5 \%) \\
15(1.2 \%)\end{array}$ & $\begin{array}{c}600(100 \%) \\
600(100 \%) \\
1200(100 \%)\end{array}$ & 0.738 \\
\hline 18 hours & $\begin{array}{c}\text { Male } \\
\text { Female } \\
\text { Total }\end{array}$ & $\begin{array}{c}6(1 \%) \\
1(0.2 \%) \\
7(0.6 \%)\end{array}$ & $\begin{array}{c}594(99 \%) \\
599(99.8 \%) \\
1193(99.4)\end{array}$ & $\begin{array}{c}600(100 \%) \\
600(100 \%) \\
1200(100 \%)\end{array}$ & 0.166 \\
\hline 24 hours & $\begin{array}{c}\text { Male } \\
\text { Female } \\
\text { Total }\end{array}$ & $\begin{array}{c}3(0.5 \%) \\
0(0 \%) \\
3(0.2 \%)\end{array}$ & $\begin{array}{c}597(99.5 \%) \\
600(100 \%) \\
1197(99.8 \%)\end{array}$ & $\begin{array}{c}600(100 \%) \\
600(100 \%) \\
1200(100 \%)\end{array}$ & 0.081 \\
\hline \multicolumn{6}{|c|}{ Distribution of subjects based on the incidence of bradycardia at different times based on gender } \\
\hline Recovery & $\begin{array}{c}\text { Male } \\
\text { Female } \\
\text { Total }\end{array}$ & $\begin{array}{l}15(2.5 \%) \\
13(2.2 \%) \\
28(2.3 \%)\end{array}$ & $\begin{array}{c}585(97.5 \%) \\
587(97.8 \%) \\
1172(97.7 \%)\end{array}$ & $\begin{array}{c}600(100 \%) \\
600(100 \%) \\
1200(100 \%)\end{array}$ & 0.827 \\
\hline 6 hours & $\begin{array}{c}\text { Male } \\
\text { Female } \\
\text { Total }\end{array}$ & $\begin{array}{l}4(0.7 \%) \\
2(0.3 \%) \\
6(0.5 \%)\end{array}$ & $\begin{array}{c}596(99.3 \%) \\
598(99.7 \%) \\
1194(99.5 \%)\end{array}$ & $\begin{array}{c}600(100 \%) \\
600(100 \%) \\
1200(100 \%)\end{array}$ & 0.693 \\
\hline 12 hours & $\begin{array}{c}\text { Male } \\
\text { Female } \\
\text { Total }\end{array}$ & $\begin{array}{l}2(0.3 \%) \\
2(0.3 \%) \\
4(0.3 \%)\end{array}$ & $\begin{array}{c}598(99.7 \%) \\
598(99.7 \%) \\
1196(99.7 \%)\end{array}$ & $\begin{array}{c}600(100 \%) \\
600(100 \%) \\
1200(100 \%)\end{array}$ & 0.738 \\
\hline 18 hours & $\begin{array}{c}\text { Male } \\
\text { Female } \\
\text { Total }\end{array}$ & $\begin{array}{l}1(0.2 \%) \\
1(0.2 \%) \\
2(0.2 \%)\end{array}$ & $\begin{array}{c}599(99.8 \%) \\
599(99.8 \%) \\
1198(99.8 \%)\end{array}$ & $\begin{array}{c}600(100 \%) \\
600(100 \%) \\
1200(100 \%)\end{array}$ & 0.166 \\
\hline
\end{tabular}




\begin{tabular}{|c|c|c|c|c|c|}
\hline 24 hours & $\begin{array}{c}\text { Male } \\
\text { Female } \\
\text { Total }\end{array}$ & $\begin{array}{c}2(0.3 \%) \\
0(0 \%) \\
2(0.2 \%)\end{array}$ & $\begin{array}{c}598(99.7 \%) \\
600(100 \%) \\
1198(99.8 \%)\end{array}$ & $\begin{array}{c}600(100 \%) \\
600(100 \%) \\
1200(100 \%)\end{array}$ & 0.081 \\
\hline \multicolumn{6}{|c|}{ Distribution of subjects based on the incidence of nausea at different times based on gender } \\
\hline Recovery & $\begin{array}{c}\text { Male } \\
\text { Female } \\
\text { Total }\end{array}$ & $\begin{array}{c}20(3.3 \%) \\
5(0.8 \%) \\
25(2 \%)\end{array}$ & $\begin{array}{c}580(99.7 \%) \\
595(99.2 \%) \\
1175(98 \%)\end{array}$ & $\begin{array}{c}600(100 \%) \\
600(100 \%) \\
1200(100 \%)\end{array}$ & 0.002 \\
\hline 6 hours & $\begin{array}{l}\text { Male } \\
\text { Female } \\
\text { Total }\end{array}$ & $\begin{array}{c}22(3.7 \%) \\
24(4 \%) \\
46(3.8 \%)\end{array}$ & $\begin{array}{c}578(96.3 \%) \\
576(96 \%) \\
1154(96.2 \%)\end{array}$ & $\begin{array}{c}600(100 \%) \\
600(100 \%) \\
1200(100 \%)\end{array}$ & 0.724 \\
\hline 12 hours & $\begin{array}{c}\text { Male } \\
\text { Female } \\
\text { Total }\end{array}$ & $\begin{array}{c}9(1.5 \%) \\
39(6.5 \%) \\
48(4 \%)\end{array}$ & $\begin{array}{c}591(98.5 \%) \\
561(93.5 \%) \\
1161(96 \%)\end{array}$ & $\begin{array}{c}600(100 \%) \\
600(100 \%) \\
1200(100 \%)\end{array}$ & 0.000 \\
\hline 18 hours & $\begin{array}{c}\text { Male } \\
\text { Female } \\
\text { Total }\end{array}$ & $\begin{array}{c}5(0.8 \%) \\
17(2.8 \%) \\
29(2.4 \%)\end{array}$ & $\begin{array}{c}595(99.2 \%) \\
583(97.2 \%) \\
1171(97.6 \%)\end{array}$ & $\begin{array}{c}600(100 \%) \\
600(100 \%) \\
1200(100 \%)\end{array}$ & 0.010 \\
\hline 24 hours & $\begin{array}{c}\text { Male } \\
\text { Female } \\
\text { Total }\end{array}$ & $\begin{array}{c}1(0.2 \%) \\
6(1 \%) \\
7(0.6 \%)\end{array}$ & $\begin{array}{c}599(99.8 \%) \\
594(99 \%) \\
1193(99.4 \%)\end{array}$ & $\begin{array}{c}600(100 \%) \\
600(100 \%) \\
1200(100 \%)\end{array}$ & 0.058 \\
\hline
\end{tabular}

\begin{tabular}{|c|c|c|c|c|c|}
\hline Recovery & $\begin{array}{c}\text { Male } \\
\text { Female } \\
\text { Total }\end{array}$ & $\begin{array}{c}595(99.2 \%) \\
600(100 \%) \\
1195(99.6 \%)\end{array}$ & $\begin{array}{l}0(0 \%) \\
0(0 \%) \\
0(0 \%)\end{array}$ & $\begin{array}{c}600(100 \%) \\
600(100 \%) \\
1200(100 \%)\end{array}$ & 0.025 \\
\hline 6 hours & $\begin{array}{c}\text { Male } \\
\text { Female } \\
\text { Total }\end{array}$ & $\begin{array}{c}588(98 \%) \\
568(94.6 \%) \\
1156(96.3 \%)\end{array}$ & $\begin{array}{l}1(0.2 \%) \\
1(0.2 \%) \\
2(0.2 \%)\end{array}$ & $\begin{array}{c}600(100 \%) \\
600(100 \%) \\
1200(100 \%)\end{array}$ & 0.007 \\
\hline 12 hours & $\begin{array}{l}\text { Male } \\
\text { Female } \\
\text { Total }\end{array}$ & $\begin{array}{l}587(97.8 \%) \\
578(96.4 \%) \\
1156(97 \%)\end{array}$ & $\begin{array}{r}1(0.2 \%) \\
2(0.3 \%) \\
3(0.3 \%)\end{array}$ & $\begin{array}{l}600(100 \%) \\
600(100 \%) \\
1200(100 \%)\end{array}$ & 0.301 \\
\hline 18 hours & $\begin{array}{c}\text { Male } \\
\text { Female } \\
\text { Total }\end{array}$ & $\begin{array}{c}593(98.8 \%) \\
584(97 \%) \\
1177(98 \%)\end{array}$ & $\begin{array}{l}1(0.2 \%) \\
4(0.7 \%) \\
5(0.4 \%)\end{array}$ & $\begin{array}{c}600(100 \%) \\
600(100 \%) \\
1200(100 \%)\end{array}$ & 0.078 \\
\hline 24 hours & $\begin{array}{l}\text { Male } \\
\text { Female } \\
\text { Total }\end{array}$ & $\begin{array}{l}594(99 \%) \\
594(99 \%) \\
1188(99 \%)\end{array}$ & $\begin{array}{l}2(0.3 \%) \\
\qquad \begin{array}{l}1(0.2 \%) \\
2(0.3 \%)\end{array}\end{array}$ & $\begin{array}{l}600(100 \%) \\
600(100 \%) \\
1200(100 \%)\end{array}$ & 0.801 \\
\hline Overall & $\begin{array}{l}\text { Male } \\
\text { Female } \\
\text { Total }\end{array}$ & $\begin{array}{c}557(92.9 \%) \\
531(88.5 \%) \\
1088(90.6 \%)\end{array}$ & $\begin{array}{c}5(0.8 \%) \\
9(1.5 \%) \\
14(1.2 \%)\end{array}$ & $\begin{array}{c}600(100 \%) \\
600(100 \%) \\
1200(100 \%)\end{array}$ & 0.006 \\
\hline
\end{tabular}

\begin{tabular}{|c|c|c|c|c|c|}
\hline \multicolumn{6}{|c|}{ Distribution of subjects based on transient neurological symptoms at different times based on gender } \\
\hline Recovery & $\begin{array}{c}\text { Male } \\
\text { Female } \\
\text { Total }\end{array}$ & $\begin{array}{l}0(0 \%) \\
0(0 \%) \\
0(0 \%)\end{array}$ & $\begin{array}{c}600(100 \%) \\
600(100 \%) \\
1200(100 \%)\end{array}$ & $\begin{array}{c}600(100 \%) \\
600(100 \%) \\
1200(100 \%)\end{array}$ & - \\
\hline 6 hours & $\begin{array}{c}\text { Male } \\
\text { Female } \\
\text { Total }\end{array}$ & $\begin{array}{l}2(0.3 \%) \\
1(0.2 \%) \\
3(0.7 \%)\end{array}$ & $\begin{array}{c}598(99.8 \%) \\
599(99.8 \%) \\
1197(99.3 \%)\end{array}$ & $\begin{array}{c}600(100 \%) \\
600(100 \%) \\
1200(100 \%)\end{array}$ & 0.563 \\
\hline 12 hours & $\begin{array}{c}\text { Male } \\
\text { Female } \\
\text { Total }\end{array}$ & $\begin{array}{l}4(0.7 \%) \\
3(0.5 \%) \\
7(0.6 \%)\end{array}$ & $\begin{array}{c}596(99.3 \%) \\
597(99.5 \%) \\
1193(99.4 \%)\end{array}$ & $\begin{array}{c}600(100 \%) \\
600(100 \%) \\
1200(100 \%)\end{array}$ & 0.705 \\
\hline 18 hours & $\begin{array}{c}\text { Male } \\
\text { Female } \\
\text { Total }\end{array}$ & $\begin{array}{l}4(0.7 \%) \\
3(0.5 \%) \\
7(0.6 \%)\end{array}$ & $\begin{array}{c}596(99.3 \%) \\
597(99.5 \%) \\
1193(99.4 \%)\end{array}$ & $\begin{array}{c}600(100 \%) \\
600(100 \%) \\
1200(100 \%)\end{array}$ & - \\
\hline 24 hours & $\begin{array}{c}\text { Male } \\
\text { Female } \\
\text { Total }\end{array}$ & $\begin{array}{l}1(0.2 \%) \\
2(0.3 \%) \\
3(0.7 \%)\end{array}$ & $\begin{array}{c}599(99.8 \%) \\
598(99.7 \%) \\
1197(99.3 \%)\end{array}$ & $\begin{array}{c}600(100 \%) \\
600(100 \%) \\
1200(100 \%)\end{array}$ & 0.563 \\
\hline \multicolumn{6}{|c|}{ Distribution of subjects based on shivering at different times based on gender } \\
\hline Recovery & $\begin{array}{c}\text { Male } \\
\text { Female } \\
\text { Total }\end{array}$ & $\begin{array}{l}16(2.7 \%) \\
19(3.2 \%) \\
35(2.9 \%)\end{array}$ & $\begin{array}{c}584(97.3 \%) \\
581(96.8 \%) \\
1165(97 \%)\end{array}$ & $\begin{array}{c}600(100 \%) \\
600(100 \%) \\
1200(100 \%)\end{array}$ & 0.607 \\
\hline 6 hours & $\begin{array}{c}\text { Male } \\
\text { Female } \\
\text { Total }\end{array}$ & $\begin{array}{c}5(0.8 \%) \\
9(1.5 \%) \\
14(1.2 \%)\end{array}$ & $\begin{array}{c}595(99.2 \%) \\
591(98.5 \%) \\
1186(98.8 \%)\end{array}$ & $\begin{array}{c}600(100 \%) \\
600(100 \%) \\
1200(100 \%)\end{array}$ & 0.282 \\
\hline
\end{tabular}




\begin{tabular}{|c|c|c|c|c|c|}
\hline 12 hours & $\begin{array}{c}\text { Male } \\
\text { Female } \\
\text { Total }\end{array}$ & $\begin{array}{c}0(0 \%) \\
1(0.2 \%) \\
1(0.008 \%)\end{array}$ & $\begin{array}{c}600(100 \%) \\
599(99.8 \%) \\
1199(99.2 \%)\end{array}$ & $\begin{array}{c}600(100 \%) \\
600(100 \%) \\
1200(100 \%)\end{array}$ & 0.317 \\
\hline 18 hours & $\begin{array}{c}\text { Male } \\
\text { Female } \\
\text { Total }\end{array}$ & $\begin{array}{l}1(0.2 \%) \\
3(0.5 \%) \\
4(0.3 \%)\end{array}$ & $\begin{array}{c}599(99.8 \%) \\
597(99.5 \%) \\
1196(99.7 \%)\end{array}$ & $\begin{array}{c}600(100 \%) \\
600(100 \%) \\
1200(100 \%)\end{array}$ & 0.317 \\
\hline 24 hours & $\begin{array}{c}\text { Male } \\
\text { Female } \\
\text { Total }\end{array}$ & $\begin{array}{l}1(0.2 \%) \\
4(0.7 \%) \\
5(0.4 \%)\end{array}$ & $\begin{array}{c}599(99.8 \%) \\
596(99.3 \%) \\
1195(99.6 \%)\end{array}$ & $\begin{array}{c}600(100 \%) \\
600(100 \%) \\
1200(100 \%)\end{array}$ & 0.179 \\
\hline
\end{tabular}

Distribution of subjects based on the incidence of low back pain at different times based on gender

\begin{tabular}{|c|c|c|c|c|c|}
\hline Recovery & $\begin{array}{c}\text { Male } \\
\text { Female } \\
\text { Total }\end{array}$ & $\begin{array}{c}0(0 \%) \\
1(0.2 \%) \\
1(0.08 \%)\end{array}$ & $\begin{array}{c}600(100 \%) \\
599(99.8 \%) \\
1199(99.92 \%)\end{array}$ & $\begin{array}{c}600(100 \%) \\
600(100 \%) \\
1200(100 \%)\end{array}$ & 0.123 \\
\hline 6 hours & $\begin{array}{c}\text { Male } \\
\text { Female } \\
\text { Total }\end{array}$ & $\begin{array}{c}4(0.7 \%) \\
9(1.5 \%) \\
13(1.1 \%)\end{array}$ & $\begin{array}{c}596(99.3 \%) \\
591(98.5 \%) \\
1187(98.9 \%)\end{array}$ & $\begin{array}{c}600(100 \%) \\
600(100 \%) \\
1200(100 \%)\end{array}$ & 0.094 \\
\hline 12 hours & $\begin{array}{c}\text { Male } \\
\text { Female } \\
\text { Total }\end{array}$ & $\begin{array}{l}2(0.3 \%) \\
7(1.2 \%) \\
9(0.5 \%)\end{array}$ & $\begin{array}{c}598(99.7 \%) \\
593(98.8 \%) \\
1191(99.5 \%)\end{array}$ & $\begin{array}{c}600(100 \%) \\
600(100 \%) \\
1200(100 \%)\end{array}$ & 0.163 \\
\hline 18 hours & $\begin{array}{c}\text { Male } \\
\text { Female } \\
\text { Total }\end{array}$ & $\begin{array}{c}0(0 \%) \\
6(1 \%) \\
6(0.5 \%)\end{array}$ & $\begin{array}{c}600(100 \%) \\
594(99 \%) \\
1194(99.5 \%)\end{array}$ & $\begin{array}{c}600(100 \%) \\
600(100 \%) \\
1200(100 \%)\end{array}$ & 0.014 \\
\hline 24 hours & $\begin{array}{c}\text { Male } \\
\text { Female } \\
\text { Total }\end{array}$ & $\begin{array}{c}1(0.2 \%) \\
6(1 \%) \\
7(0.6 \%)\end{array}$ & $\begin{array}{c}599(99.8 \%) \\
594(99 \%) \\
1193(99.4 \%)\end{array}$ & $\begin{array}{c}600(100 \%) \\
600(100 \%) \\
1200(100 \%)\end{array}$ & 0.058 \\
\hline
\end{tabular}

Distribution of the subjects on the basis of restlessness at different times based on gender

\begin{tabular}{|c|c|c|c|c|c|}
\hline Recovery & $\begin{array}{c}\text { Male } \\
\text { Female } \\
\text { Total }\end{array}$ & $\begin{array}{l}1(0.2 \%) \\
2(0.3 \%) \\
3(0.2 \%)\end{array}$ & $\begin{array}{c}599(99.8 \%) \\
598(99.7 \%) \\
1197(99.8 \%)\end{array}$ & $\begin{array}{c}600(100 \%) \\
600(100 \%) \\
1200(100 \%)\end{array}$ & 0.563 \\
\hline 6 hours & $\begin{array}{c}\text { Male } \\
\text { Female } \\
\text { Total }\end{array}$ & $\begin{array}{l}2(0.3 \%) \\
3(0.5 \%) \\
5(0.4 \%)\end{array}$ & $\begin{array}{c}598(99.5 \%) \\
597(99.5 \%) \\
1195(99.6 \%)\end{array}$ & $\begin{array}{c}600(100 \%) \\
600(100 \%) \\
1200(100 \%)\end{array}$ & 0.654 \\
\hline 12 hours & $\begin{array}{c}\text { Male } \\
\text { Female } \\
\text { Total }\end{array}$ & $\begin{array}{l}0(0 \%) \\
0(0 \%) \\
0(0 \%)\end{array}$ & $\begin{array}{c}600(100 \%) \\
600(100 \%) \\
1200(100 \%)\end{array}$ & $\begin{array}{c}600(100 \%) \\
600(100 \%) \\
1200(100 \%)\end{array}$ & - \\
\hline 18 hours & $\begin{array}{c}\text { Male } \\
\text { Female } \\
\text { Total }\end{array}$ & $\begin{array}{l}4(0.7 \%) \\
5(0.8 \%) \\
9(0.7 \%)\end{array}$ & $\begin{array}{c}596(99.3 \%) \\
595(99.2 \%) \\
1191(99.3 \%)\end{array}$ & $\begin{array}{c}600(100 \%) \\
600(100 \%) \\
1200(100 \%)\end{array}$ & 0.738 \\
\hline 24 hours & $\begin{array}{c}\text { Male } \\
\text { Female } \\
\text { Total }\end{array}$ & $\begin{array}{c}1(0.2 \%) \\
0(0 \%) \\
1(0.08 \%)\end{array}$ & $\begin{array}{c}599(99.8 \%) \\
600(100 \%) \\
1199(99.92 \%)\end{array}$ & $\begin{array}{c}600(100 \%) \\
600(100 \%) \\
1200(100 \%)\end{array}$ & 0.317 \\
\hline
\end{tabular}

Distribution of subjects based on the incidence of urinary retention at different times based on gender

\begin{tabular}{|c|c|c|c|c|c|}
\hline Recovery & $\begin{array}{c}\text { Male } \\
\text { Female } \\
\text { Total }\end{array}$ & $\begin{array}{l}32(5.3 \%) \\
13(2.2 \%) \\
45(3.8 \%)\end{array}$ & $\begin{array}{c}568(94.7 \%) \\
587(97.8 \%) \\
1100(96.3 \%)\end{array}$ & $\begin{array}{c}600(100 \%) \\
600(100 \%) \\
1200(100 \%)\end{array}$ & 0.004 \\
\hline 6 hours & $\begin{array}{c}\text { Male } \\
\text { Female } \\
\text { Total }\end{array}$ & $\begin{array}{c}26(4.3 \%) \\
36(6 \%) \\
62(5.2 \%)\end{array}$ & $\begin{array}{c}574(95.7 \%) \\
464(94 \%) \\
1138(94.8 \%)\end{array}$ & $\begin{array}{c}600(100 \%) \\
600(100 \%) \\
1200(100 \%)\end{array}$ & 0.192 \\
\hline 12 hours & $\begin{array}{c}\text { Male } \\
\text { Female } \\
\text { Total }\end{array}$ & $\begin{array}{c}3(0.5 \%) \\
7(1.2 \%) \\
10(0.8 \%)\end{array}$ & $\begin{array}{c}597(98.5 \%) \\
593(98.8 \%) \\
1190(99.2 \%)\end{array}$ & $\begin{array}{c}600(100 \%) \\
600(100 \%) \\
1200(100 \%)\end{array}$ & 0.204 \\
\hline 18 hours & $\begin{array}{c}\text { Male } \\
\text { Female } \\
\text { Total }\end{array}$ & $\begin{array}{c}7(1.2 \%) \\
4(0.7 \%) \\
11(0.9 \%)\end{array}$ & $\begin{array}{c}593(99.8 \%) \\
596(99.3 \%) \\
1189(99.1 \%)\end{array}$ & $\begin{array}{c}600(100 \%) \\
600(100 \%) \\
1200(100 \%)\end{array}$ & 0.364 \\
\hline 24 hours & $\begin{array}{c}\text { Male } \\
\text { Female } \\
\text { Total }\end{array}$ & $\begin{array}{c}5(0.8 \%) \\
0(0 \%) \\
5(0.4 \%)\end{array}$ & $\begin{array}{c}595(99.2 \%) \\
600(100 \%) \\
1190(99.6 \%)\end{array}$ & $\begin{array}{c}600(100 \%) \\
600(100 \%) \\
1200(100 \%)\end{array}$ & 0.075 \\
\hline
\end{tabular}

Distribution of subjects based on the incidence of hypothermia at different times based on gender

\begin{tabular}{|c|c|c|c|c|c|}
\hline & Male & $17(2.8 \%)$ & $583(97.2 \%)$ & $600(100 \%)$ & \\
Recovery & Female & $10(1.7 \%)$ & $590(98.3 \%)$ & $600(100 \%)$ & 0.173 \\
& Total & $27(2.2 \%)$ & $1173(98.8 \%)$ & $1200(100 \%)$ & \\
\hline
\end{tabular}




\begin{tabular}{|c|c|c|c|c|c|}
\hline 6 hours & $\begin{array}{c}\text { Male } \\
\text { Female } \\
\text { Total }\end{array}$ & $\begin{array}{l}3(0.5 \%) \\
1(0.2 \%) \\
4(0.3 \%)\end{array}$ & $\begin{array}{c}597(99.5 \%) \\
599(99.8 \%) \\
1196(99.7 \%)\end{array}$ & $\begin{array}{c}600(100 \%) \\
600(100 \%) \\
1200(100 \%)\end{array}$ & 0.968 \\
\hline 12 hours & $\begin{array}{c}\text { Male } \\
\text { Female } \\
\text { Total }\end{array}$ & $\begin{array}{c}0(0 \%) \\
3(0.5 \%) \\
3(0.2 \%)\end{array}$ & $\begin{array}{c}600(100 \%) \\
597(99.5 \%) \\
1197(99.8 \%)\end{array}$ & $\begin{array}{c}600(100 \%) \\
600(100 \%) \\
1200(100 \%)\end{array}$ & 0.083 \\
\hline 18 hours & $\begin{array}{l}\text { Male } \\
\text { Female } \\
\text { Total }\end{array}$ & $\begin{array}{c}0(0 \%) \\
5(0.8 \%) \\
5(0.4 \%)\end{array}$ & $\begin{array}{c}600(100 \%) \\
595(99.2 \%) \\
1195(99.6 \%)\end{array}$ & $\begin{array}{c}600(100 \%) \\
600(100 \%) \\
1200(100 \%)\end{array}$ & 0.055 \\
\hline 24 hours & $\begin{array}{c}\text { Male } \\
\text { Female } \\
\text { Total }\end{array}$ & $\begin{array}{c}0(0 \%) \\
2(0.3 \%) \\
2(0.2 \%)\end{array}$ & $\begin{array}{c}600(100 \%) \\
598(99.7 \%) \\
1198(99.8 \%)\end{array}$ & $\begin{array}{c}600(100 \%) \\
600(100 \%) \\
1200(100 \%)\end{array}$ & 0.157 \\
\hline \multicolumn{6}{|c|}{ Distribution of subjects based on bradypenea at different times based on gender } \\
\hline Recovery & $\begin{array}{l}\text { Male } \\
\text { Female } \\
\text { Total }\end{array}$ & $\begin{array}{l}3(0.5 \%) \\
4(0.7 \%) \\
7(0.6 \%)\end{array}$ & $\begin{array}{c}597(99.5 \%) \\
596(99.3 \%) \\
1193(96.4 \%)\end{array}$ & $\begin{array}{c}600(100 \%) \\
600(100 \%) \\
1200(100 \%)\end{array}$ & 0.899 \\
\hline 6 hours & $\begin{array}{c}\text { Male } \\
\text { Female } \\
\text { Total }\end{array}$ & $\begin{array}{l}0(0 \%) \\
0(0 \%) \\
0(0 \%)\end{array}$ & $\begin{array}{c}600(100 \%) \\
600(100 \%) \\
1200(100 \%)\end{array}$ & $\begin{array}{c}600(100 \%) \\
600(100 \%) \\
1200(100 \%)\end{array}$ & - \\
\hline 12 hours & $\begin{array}{c}\text { Male } \\
\text { Female } \\
\text { Total }\end{array}$ & $\begin{array}{l}0(0 \%) \\
0(0 \%) \\
0(0 \%)\end{array}$ & $\begin{array}{c}600(100 \%) \\
600(100 \%) \\
1200(100 \%)\end{array}$ & $\begin{array}{c}600(100 \%) \\
600(100 \%) \\
1200(100 \%)\end{array}$ & - \\
\hline 18 hours & $\begin{array}{c}\text { Male } \\
\text { Female } \\
\text { Total }\end{array}$ & $\begin{array}{c}0(0 \%) \\
2(0.3 \%) \\
2(0.2 \%)\end{array}$ & $\begin{array}{c}600(100 \%) \\
600(99.7 \%) \\
1198(99.8 \%)\end{array}$ & $\begin{array}{c}600(100 \%) \\
600(100 \%) \\
1200(100 \%)\end{array}$ & 0.217 \\
\hline 24 hours & $\begin{array}{c}\text { Male } \\
\text { Female } \\
\text { Total }\end{array}$ & $\begin{array}{l}4(0.3 \%) \\
0(0 \%) \\
0(0 \%)\end{array}$ & $\begin{array}{c}0(0 \%) \\
0(0 \%) \\
1200(100 \%)\end{array}$ & $\begin{array}{c}600(100 \%) \\
600(100 \%) \\
1200(100 \%)\end{array}$ & - \\
\hline
\end{tabular}

Table 3: Distribution of subjects based on the incidence of different parameters at different times based on gender

\begin{tabular}{|c|c|c|c|c|c|c|c|}
\hline Time & Group & $\begin{array}{l}\text { No headache } \\
\text { (percent) }\end{array}$ & $\begin{array}{l}\text { Mild headache } \\
\text { (percent) }\end{array}$ & $\begin{array}{l}\text { Average } \\
\text { headache } \\
\text { (percent) }\end{array}$ & $\begin{array}{c}\text { Severe } \\
\text { headache } \\
\text { (percent) }\end{array}$ & $\begin{array}{c}\text { Total } \\
\text { Number } \\
\text { (percent) }\end{array}$ & P-value \\
\hline Recovery & $\begin{array}{c}\text { Male } \\
\text { Female } \\
\text { Total }\end{array}$ & $\begin{array}{c}598(99.7 \%) \\
599(99.8 \%) \\
1197(99.7 \%)\end{array}$ & $\begin{array}{l}1(0.2 \%) \\
1(0.2 \%) \\
2(0.2 \%)\end{array}$ & $\begin{array}{c}1(0.2 \%) \\
0(0 \%) \\
1(1 \%)\end{array}$ & $\begin{array}{l}0(0 \%) \\
0(0 \%) \\
0(0 \%)\end{array}$ & $\begin{array}{c}600(100 \%) \\
600(100 \%) \\
1200(100 \%)\end{array}$ & 0.606 \\
\hline 6 hours & $\begin{array}{c}\text { Male } \\
\text { Female } \\
\text { Total }\end{array}$ & $\begin{array}{c}598(99.7 \%) \\
596(99.3 \%) \\
1194(99.5 \%)\end{array}$ & $\begin{array}{l}2(0.3 \%) \\
4(0.7 \%) \\
6(0.5 \%)\end{array}$ & $\begin{array}{l}0(0 \%) \\
0(0 \%) \\
0(0 \%)\end{array}$ & $\begin{array}{l}0(0 \%) \\
0(0 \%) \\
0(0 \%)\end{array}$ & $\begin{array}{c}600(100 \%) \\
600(100 \%) \\
1200(100 \%)\end{array}$ & 0.413 \\
\hline 12 hours & $\begin{array}{c}\text { Male } \\
\text { Female } \\
\text { Total }\end{array}$ & $\begin{array}{c}591(99.5 \%) \\
592(98.7 \%) \\
1183(98.6 \%)\end{array}$ & $\begin{array}{c}8(1.3 \%) \\
7(1.2 \%) \\
15(1.2 \%)\end{array}$ & $\begin{array}{c}1(0.2 \%) \\
0(0 \%) \\
1(0.1 \%)\end{array}$ & $\begin{array}{c}0(0 \%) \\
1(0.2 \%) \\
1(0.1 \%)\end{array}$ & $\begin{array}{c}600(100 \%) \\
600(100 \%) \\
1200(100 \%)\end{array}$ & 0.599 \\
\hline 18 hours & $\begin{array}{c}\text { Male } \\
\text { Female } \\
\text { Total }\end{array}$ & $\begin{array}{c}592(98.7 \%) \\
573(95.5 \%) \\
1165(97.1 \%)\end{array}$ & $\begin{array}{c}4(0.7 \%) \\
22(3.7 \%) \\
26(2.2 \%)\end{array}$ & $\begin{array}{l}4(0.7 \%) \\
1(0.2 \%) \\
5(0.4 \%)\end{array}$ & $\begin{array}{c}0(0 \%) \\
4(0.7 \%) \\
4(0.3 \%)\end{array}$ & $\begin{array}{c}600(100 \%) \\
600(100 \%) \\
1200(100 \%)\end{array}$ & 0.559 \\
\hline 24 hours & $\begin{array}{c}\text { Male } \\
\text { Female } \\
\text { Total }\end{array}$ & $\begin{array}{l}589(98.2 \%) \\
574(95.7 \%) \\
1163(97 \%)\end{array}$ & $\begin{array}{c}6(1 \%) \\
19(3.2 \%) \\
25(2.1 \%)\end{array}$ & $\begin{array}{l}2(0.3 \%) \\
1(0.2 \%) \\
3(0.2 \%)\end{array}$ & $\begin{array}{c}3(0.5 \%) \\
6(1 \%) \\
9(0.7 \%)\end{array}$ & $\begin{array}{c}600(100 \%) \\
600(100 \%) \\
1200(100 \%)\end{array}$ & 0.040 \\
\hline Second day & $\begin{array}{c}\text { Male } \\
\text { Female } \\
\text { Total }\end{array}$ & $\begin{array}{l}590(98.3 \%) \\
586(97.7 \%) \\
1176(98 \%)\end{array}$ & $\begin{array}{c}7(1.2 \%) \\
9(1.5 \%) \\
16(1.3 \%)\end{array}$ & $\begin{array}{l}3(0.5 \%) \\
5(0.8 \%) \\
8(0.7 \%)\end{array}$ & $\begin{array}{l}0(0 \%) \\
0(0 \%) \\
0(0 \%)\end{array}$ & $\begin{array}{c}600(100 \%) \\
600(100 \%) \\
1200(100 \%)\end{array}$ & 0.683 \\
\hline Third day & $\begin{array}{c}\text { Male } \\
\text { Female } \\
\text { Total }\end{array}$ & $\begin{array}{c}590(98.3 \%) \\
589(98.2 \%) \\
1179(98.2 \%)\end{array}$ & $\begin{array}{c}8(1.3 \%) \\
9(1.5 \%) \\
17(1.4 \%)\end{array}$ & $\begin{array}{l}2(0.3 \%) \\
2(0.3 \%) \\
4(0.3 \%)\end{array}$ & $\begin{array}{l}0(0 \%) \\
0(0 \%) \\
0(0 \%)\end{array}$ & $\begin{array}{c}600(100 \%) \\
600(100 \%) \\
1200(100 \%)\end{array}$ & 0.971 \\
\hline Overall & $\begin{array}{c}\text { Male } \\
\text { Female } \\
\text { Total }\end{array}$ & $\begin{array}{c}560(93.3 \%) \\
522(87 \%) \\
1082(90.1 \%)\end{array}$ & $\begin{array}{c}28(4.7 \%) \\
61(10.2 \%) \\
89(7.4 \%)\end{array}$ & $\begin{array}{c}9(1.5 \%) \\
6(1 \%) \\
10(1.3 \%)\end{array}$ & $\begin{array}{c}3(0.5 \%) \\
11(1.8 \%) \\
14(1.2 \%)\end{array}$ & $\begin{array}{c}600(100 \%) \\
600(100 \%) \\
1200(100 \%)\end{array}$ & 0.000 \\
\hline
\end{tabular}

Table 4: Distribution of subjects based on the occurrence of headache and headache intensity at different times based on gender

The incidence of severe pain in men and women was $5(0.8 \%)$ respectively in the surgical area, but, men did not complain of severe pain at the surgical site, there was no significant difference observed between incidence of surgical pain and gender $(\mathrm{P}=0.245)$ (Table 5). Pain observed during the injection of anesthetic was $5(0.8 \%)$ in men and $6(1 \%)$ in women, there was no significant difference observed between gender and pains while injecting a local anesthesia $(\mathrm{P}=0.762)$ (Table 6). 


\begin{tabular}{|c|c|c|c|c|c|c|c|}
\hline Time & Group & No pain & Mild pain & $\begin{array}{c}\text { Average pain } \\
\text { (percent) }\end{array}$ & $\begin{array}{c}\text { Severe pain } \\
\text { (percent) }\end{array}$ & $\begin{array}{c}\text { Total Number } \\
\text { (percent) }\end{array}$ & P-value \\
\hline \multirow{5}{*}{ Recovery } & Male & $599(99.8 \%)$ & $1(0.2 \%)$ & $0(0 \%)$ & $0(0 \%)$ & $600(100 \%)$ & \\
& Female & $597(99.3 \%)$ & $3(0.5 \%)$ & $0(0 \%)$ & $0(0 \%)$ & $600(100 \%)$ & 0.366 \\
& Total & $1196(99.7 \%)$ & $4(0.3 \%)$ & $0(0 \%)$ & $0(0 \%)$ & $1200(100 \%)$ & \\
\hline \multirow{4}{*}{6 hours } & Male & $589(98.2 \%)$ & $11(1.8 \%)$ & $0(0 \%)$ & $0(0 \%)$ & $600(100 \%)$ & \\
& Female & $586(97.5 \%)$ & $13(2.2 \%)$ & $1(0.2 \%)$ & $0(0 \%)$ & $600(100 \%)$ & 0.536 \\
& Total & $1175(98 \%)$ & $24(2 \%)$ & $1(0.1 \%)$ & $0(0 \%)$ & $1200(100 \%)$ & \\
\hline \multirow{4}{*}{12 hours } & Male & $591(98.5 \%)$ & $8(1.3 \%)$ & $1(0.2 \%)$ & $0(0 \%)$ & $600(100 \%)$ & \\
& Female & $588(98 \%)$ & $6(1 \%)$ & $3(0.5 \%)$ & $3(0.5 \%)$ & $600(100 \%)$ & 0.231 \\
& Total & $1179(98.3 \%)$ & $14(1.2 \%)$ & $4(0.3 \%)$ & $3(0.2 \%)$ & $1200(100 \%)$ & \\
\hline \multirow{4}{*}{24 hours hours } & Male & $595(99.2 \%)$ & $3(0.5 \%)$ & $2(0.3 \%)$ & $0(0 \%)$ & $600(100 \%)$ & \\
& Female & $596(99.2 \%)$ & $0(0 \%)$ & $3(0.5 \%)$ & $1(0.2 \%)$ & $600(100 \%)$ & 0.241 \\
& Total & $1191(99.3 \%)$ & $3(0.2 \%)$ & $5(0.4 \%)$ & $1(0.1 \%)$ & $1200(100 \%)$ & \\
\hline \multirow{3}{*}{ Overall } & Male & $597(99.5 \%)$ & $3(0.5 \%)$ & $0(0 \%)$ & $0(0 \%)$ & $600(100 \%)$ & \\
& Female & $599(99.8 \%)$ & $0(0 \%)$ & $0(0 \%)$ & $1(0.2 \%)$ & $600(100 \%)$ & 0.135 \\
& Total & $1196(99.7 \%)$ & $3(0.2 \%)$ & $0(0 \%)$ & $1(0.1 \%)$ & $1200(100 \%)$ & \\
& Male & $570(95 \%)$ & $26(4.3 \%)$ & $4(0.7 \%)$ & $0(0 \%)$ & $600(100 \%)$ & \\
& Female & $568(94.7 \%)$ & $22(3.7 \%)$ & $7(1.2 \%)$ & $5(0.8 \%)$ & $600(100 \%)$ & 0.240 \\
& Total & $1138(94.8 \%)$ & $48(4 \%)$ & $11(0.8 \%)$ & $5(0.4 \%)$ & $1200(100 \%)$ & \\
\hline
\end{tabular}

Table 5. Distribution of subjects based on pain and surgical pain postoperatively at different times based on gender

\begin{tabular}{|c|c|c|c|c|c|}
\hline Variable & Group & Number present & Mild pain & Total Number (percent) & P-value \\
\hline \multirow{2}{*}{$\begin{array}{c}\text { Pain during } \\
\text { anesthesia }\end{array}$} & Men & $595(99.2) \%$ & $5(0.8) \%$ & $600(100 \%)$ & \\
\cline { 2 - 5 } & Women & $594(99) \%$ & $6(1) \%$ & $600(100 \%)$ & \multirow{2}{*}{0.762} \\
\cline { 2 - 5 } & Total & $1189(99.1 \%)$ & $11(0.9 \%)$ & $1200(100 \%)$ & \\
\hline
\end{tabular}

Table 6. Distribution of subjects based on the occurrence of pain during injection numbness and pain intensity at different times based on gender

\section{Discussion and Conclusion}

The present study examined the relationship between gender and adverse effects after spinal anesthesia with lidocaine. In this study, statistical analysis of the 19 variables studied showed a significant difference between the incidence of 5 variables and gender of patients. These variables include vomiting, nausea, headache, urinary retention and back pain after spinal anesthesia [13]. The incidence of nausea in men and women was 57 patients $(9.5 \%)$ and $91(15.2 \%)$ respectively $(\mathrm{P}=0.004)$. The incidence of vomiting in men and women was $34(6.3 \%)$, and $60(10 \%)$, also average vomiting in men and women was 5 patients $(0.8 \%)$ and 9 patients (1.5\%) (with severe vomiting) respectively, there was a statistically significantly difference observed between incidence of vomiting and gender $(\mathrm{P}=0.006)$. In a meta-analysis study conducted by Apfel et al. [14], they found that female gender can be a risk factor for nausea and vomiting following spinal anesthesia. In another study conducted by Koivuranata et al. nausea and vomiting are the most important, and most common complications introduced [15]. Risk factors such as gender, gynecologic surgery or duration of surgery more than 60 minutes can cause this complications. In a study by Quinn et al. incidence of PONV in women and men were $46 \%$ and $26 \%$ respectively [16]. Sinclair et al. in a study of the incidence of nausea found that, it was $28 \%$ and $14 \%$ in women and men respectively, $17 \%$ and $7 \%$ incidence of vomiting was reported in women and men respectively [17]. Among the factors that could lead to an increasing incidence of this complication in the female gender is the menstrual cycle [18]. In a study conducted by Vanden et al. they found that there is mutual relationship between anxiety and the incidence of PONV after surgery [19]. Since restlessness is more common in women, incidence of PONV can be higher [20]. In the present study, the incidence of mild pain in women and men was $10.2 \%$ and $4.7 \%$ respectively, the occurrence of average headache average was $1 \%$ and $1.5 \%$ in women and men respectively, the incidence of severe headache in women and men were $1.8 \%$ and $0.5 \%$, respectively. As a result, there was a significant relationship between gender and headache $(\mathrm{P}<0.05)$.

In a study conducted by Dripps et al. the incidence of headache in women was twice that of men which are $14 \%$ and $7 \%$ respectively [21]. In another study by Spencer et al., the incidence of headache in women and men was 12\% and 7\% respectively. Kang et al. found the occurrence of headache in women and men to be $13.4 \%$ and $5.7 \%$ respectively, suggesting that sex can be a crucial factor in the development of headache, there was a similarity between these studies and the present study, showing a direct relationship between sexes, nausea, vomiting and headache [22,23]. There are several factors accounting for this difference between the sexes expressed. One of these causes more tension headaches and migraines among women [24]. Conversely, women may be more sensitive to experimental pain tolerance than men [25]. Among the causes, sex hormones can also increase the incidence of this complication among women [26].

The incidence of back pain in men and women was 7 patients (1.2\%) and $14(2.3 \%)$ respectively, there was a statistically significantly difference observed between back pain and gender $(\mathrm{P}=0.313)$, but, significant difference was observed 18 hours after surgery $(\mathrm{P}=0.014)$ [27]. It should be noted that different factors accounts for back pain after spinal anesthesia [28]. Previous history of the 
cause of back pain include; how the needles are inserted for spinal anesthesia, muscle strength presented, and risk factors for lower back pain [29]. Perhaps because women have less muscle mass, tendons becomes more flexible due to the presence of female sex hormones and lumbar lordosis, which accounts for more pain they are experiencing [30].

The incidence of urinary retention in men and women was $73(12.2 \%)$, and 60 (10\%) respectively, there was a statistically significantly difference observed between urinary retention and gender $(\mathrm{P}<0.05)$. In a study by Keita et al. $(14)$, as well as Stallard et al., about comparison between gender and incidence of urinary retention, there was no significant difference observed. According to the survey conducted by Axelsson et al., and Tammela et al., sex can be considered a risk factor for the occurrence of headache. This may explain the significant relationship between genders and increase incidence of urinary retention upon recovery [31-34]. Most men do had non-urological surgery after experiencing urinary retention, and the incidence is also high. It is better to know that the main cause of many cases of hypertrophy is not prostatic [35]. It causes an imbalance in the mechanisms and structure of the male body, effects of several kinds of surgery, anesthesia and post-operative care is different [36]. Usually people urinates after surgery until they have to spend time. These people are not able to move due to multiple IV routes. If you can't urinate when lying down or sitting on the bed. Privacy to urinate is often absent in many hospitals which itself may be the delay in urination. When you want to urinate due to pain, especially the lower abdominal pain, muscle relaxes, and prevents the reflux of urine, this can cause pain to start. The rapid infusion of IV fluids during and after surgery led to the fast filling of the bladder [37]. All these factors cause a greater retention in male urine [38]. So it is better for men in recovery, to be attracted with the most attention. Because usually men due to their physiological and psychological structure creates more resistant to these complications and may complain, worsening the condition and creating a vicious cycle. According to this study, gender can give rise to differences in the incidence of some complications after spinal anesthesia of lidocaine, hence, it must therefore be resolved. Incidence of this complication can be predicted, leading to a better management of patients after the surgery.

\section{Acknowledgement}

Financial support was offered by Lorestan University of Medical Sciences, Khoramabad. Iran

\section{Conflict of interest}

The authors have no conflict of interest

\section{References}

1. Vahabi S, Heidari M, Ahmadinejad M, Akhlaghi J, Birjandi M (2011) Comparison of local anesthetic effects of tramadol and lidocaine used subcutaneously in minor surgeries with local anesthesia. Middle East J Anesthesiol 21: 9-14.

2. Craig CM, Nejim J (2016) Regional Anesthesia Hospital. Medicine Clinics 5: 71-84.

3. Fettes PDW, Jansson JR, Wildsmith JA (2009) Failed spinal anaesthesia: mechanisms, management, and prevention. Br J Anaesth 102: 739-48.

4. Vahabi S, Nadri S, Izadi F (2014) The effects of gabapentin on severity of post spinal anesthesia headache. Pak J Pharm Sci 27: 1203-7.

5. Reddy S, Weinberg L, Young P (2016) Crystalloid fluid therapy. Crit Care 20: 59.

6. Vahabi S, Eatemadi A (2017) Effects of anesthetic and analgesic techniques on cancer metastasis. Biomed Pharmacother 87: 1-7.

7. Ruppert V, Leurs LJ, Steckmeier B, Buth J, Umscheid T (2006) Influence of anesthesia type on outcome after endovascular aortic aneurysm repair: An analysis based on EUROSTAR data. J Vasc Surg 44: 16-21.

8. Lacasse MA, Roy JD, Forget J, Vandenbroucke F, Seal RF, et al. (2011) Comparison of bupivacaine and 2-chloroprocaine for spinal anesthesia for outpatient surgery: A double-blind randomized trial. Can J Anesth 58: 384-91.

9. Miron R (2007) The Washington Manual of Medical Therapeutics. Acta Endocrinologica (Bucharest) 3: 531-531.

10. Dadarkar P, Philip J, Weidner C, Perez B, Slaymaker E (2004) Spinal anesthesia for cesarean section following inadequate labor epidural analgesia: A retrospective audit. Int J Obstet Anesth 13: 239-43.

11. Nadri S, Mahmoudvand H, Vahabi S (2017) Effect of magnesium sulfate on morphine activity retention to control pain after herniorrhaphy. Pain Manag 7 : 119-25.

12. Apfel CC, Heidrich FM, Jukar-Rao S, Jalota L, Hornuss C, et al. (2012) Evidence-based analysis of risk factors for postoperative nausea and vomiting. Br J Anaesth 109: 742-53.

13. Vahabi S, Rafieian Y, Abbas Zadeh A (2018) The Effects of Intraoperative Esmolol Infusion on the Postoperative Pain and Hemodynamic Stability after Rhinoplasty. J Investig Surg 31: 82-8.

14. Apfel CC, Läärä E, Koivuranta M, Greim CA, Roewer N (1999) A simplified risk score for predicting postoperative nausea and vomiting: conclusions from cross-validations between two centers. Anesthesiology 91: 693-700.

15. Koivuranta M, Läärä E, Snåre L, Alahuhta S (1997) A survey of postoperative nausea and vomiting. Anaesthesia 52: 443-9.

16. Quinn AC, Brown JH, Wallace PG, Asbury AJ (1994) Studies in postoperative sequelae. Nausea and vomiting???still a problem. Anaesthesia 49: 62-5.

17. Sinclair DR, Chung F, Mezei G (1999) Can postoperative nausea and vomiting be predicted? Anesthesiology 91:109-18.

18. Beiranvand S, Vahabi S (2017) Effect of Local Ropivacaine on Hemodynamic Responses in Craniotomy Patients. J Investig Surg 2017 : 1-5.

19. Van den Bosch JE, Moons KG, Bonsel GJ, Kalkman CJ (2005) Does measurement of preoperative anxiety have added value for predicting postoperative nausea and vomiting? Anesth Analg 100: 1525-32.

20. Vahabi S, Eatemadi A (2016) Phyto-anesthetics: A mini-review on herb-anesthesia drug interactions. Biomed Pharmacother 84: 1885-90.

21. Dripps RD, Vandam LD (1954) Long-term follow-up of patients who received 10,098 spinal anesthetics: failure to discover major neurological sequelae. J Am Med Assoc 156: 1486-91. 
22. Kang SB, Goodnough DE, Lee YK, Olson RA, Borshoff JA (1992) Comparison of 26- and 27-G needles for spinal anesthesia for ambulatory surgery patients. Anesthesiology 76: 734-8.

23. Beiranvand S, Eatemadi A, Karimi A (2016) New Updates Pertaining to Drug Delivery of Local Anesthetics in Particular Bupivacaine Using Lipid Nanoparticles. Nanoscale Res Lett 11: 1-10.

24. Beiranvand S, Moradkhani MR (2017) Bupivacaine Versus Liposomal Bupivacaine For Pain Control. Drug Res (Stuttg) 2017: doi: 10.1055/s-0043-121142.

25. Moradkhani MR, Karimi A (2017) Role of Drug Anesthesia and Cancer. Drug Res (Stuttg) 68: 125-31.

26. Hassanzadeh-Kiabi F, Negahdari B (2018) Antinociceptive synergistic interaction between Achillea millefolium and Origanum vulgare L. extract encapsulated in liposome in rat. Artif Cells Nanomed Biotechnol 46:994-1000.

27. Moradkhani MR, Karimi A, Negahdari B (2018) Nanotechnology application to local anaesthesia (LA). Artif Cells Nanomed Biotechnol 46: 355-60.

28. Moradkhani MR, Karimi A, Negahdari B (2018) Nanotechnology application for pain therapy. Artif Cells Nanomed Biotechnol 46: $368-73$.

29. Momenzadeh S, Moradkhani M, Moghadam MH (2012) Low Level Laser Therapy (LLLT) Combined with Physical Exercise, A More Effective Treatment in Low Back Pain. J Lasers Med. Sci 3: 67.

30. Beiranvand S, Karimi A (2018) Effect of Encapsulated Artemisia aucheri. L Magnetic Nanogel Extract on Shoulder Block in Rat. Drug Res (Stuttg) 68: 65-71.

31. Stallard S, Prescott S (1988) Postoperative urinary retention in general surgical patients. Br. J. Surg 75: 1141-3.

32. Axelsson K, Möllefors K, Olsson JO, Lingårdh G, Widman B (1985) Bladder Function in Spinal Anaesthesia. Acta Anaesthesiol Scand 29: $315-21$.

33. Tammela T, Kontturi M, Lukkarinen O (1986) Postoperative urinary retention: I. Incidence and predisposing factors. Scand J Urol Nephrol 20 : $197-201$.

34. Hasanzadeh-Kiabi F (2018) Nano-drug for Pain Medicine. Drug Res (Stuttg) 68: 245-9.

35. Nadri S, Mahmoudvand H, Rokrok S, Tarrahi MJ (2017) Comparison of Two Methods: Spinal Anesthesia and Ischiorectal Block on Post Hemorrhoidectomy Pain and Hospital Stay: A Randomized Control Trial. J Invest Surg 2017: 1-5.

36. Hasanzadeh-kiabi F, Negahdari B (2017) Applications of Drug Anesthesia in Control Chronic Pain. J Investig Surg: 1-6.

37. Ahmadvand H, Shahsavari G, Tavafi M, Bagheri S, Moradkhani MR, et al. (2017) Protective effects of oleuropein against renal injury oxidative damage in alloxan-induced diabetic rats; a histological and biochemical study. J Nephropathol 6: 204-9.

38. Mardani M, Mahmoud B, Moradmand JS, Salehi A, Davoodi M, et al. (2016) Comparison of the descurainia sophia and levostatin effect on the ldl cholesterol reduction, a clinical trial study. J Chem Pharm Sci 9: 1329-33.

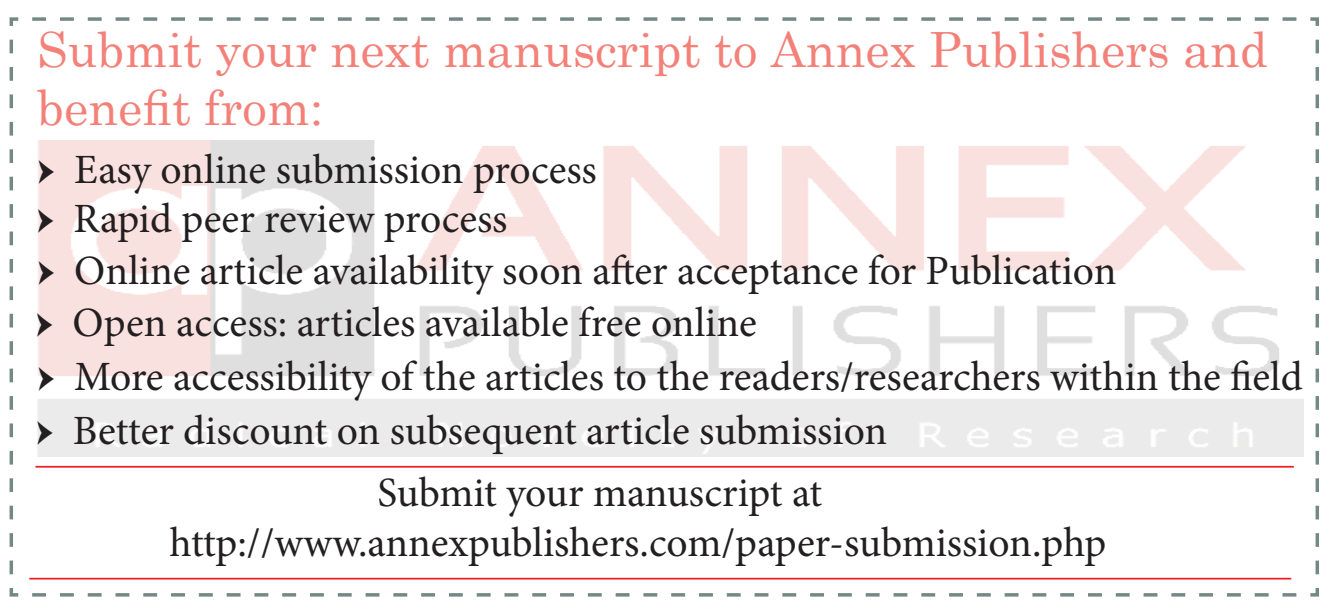

